\title{
Velocity dependence of kinetic friction in the Prandtl-Tomlinson model
}

\author{
Martin H. Müser \\ Jülich Supercomputer Centre, Institute for Advanced Simulation, FZ Jülich, Jülich, Germany and \\ Department of Materials Science and Engineering, Universität des Saarlandes, Saarbrücken, Germany
}

(Received 7 July 2011; published 8 September 2011)

\begin{abstract}
The Prandtl-Tomlinson model for friction has been used extensively for the interpretation of atomic force microscopy data during the past decade. Up to this point, the kinetic friction $F_{k}$ has nevertheless not been studied in a range of velocities $v$ that would be sufficiently broad to cover the crossover from the high-velocity logarithmic to the low-velocity linear $F_{k}(v)$ dependence. This gap will be closed here through a combination of an asymptotic analysis and direct simulations of the relevant Langevin equation. The simulations span three decades in temperature $T$ and up to six decades in $v$. All numerical data can be fit quite accurately with a $F_{k}=a(T) \operatorname{arsinh}\left[v / v_{\mathrm{c}}(T)\right]$ law, where the prefactor $a(T)$ scales with $T^{2 / 3}$. Correction terms proportional to odd powers of $\operatorname{arsinh}\left(v / v_{\mathrm{c}}\right)$, only need to be included at $v \gg v_{\mathrm{c}}$. Reasons are given as to why it is difficult to confirm meticulously the $(\ln v)^{2 / 3}$ dependence of kinetic friction predicted by recent rate theories, although they can be easily modified to produce the correct prefactor to the $a(T) \propto T^{2 / 3}$ law.
\end{abstract}

DOI: 10.1103/PhysRevB.84.125419

PACS number(s): 68.35.Af, 46.55.+d, 81.40.Pq

\section{INTRODUCTION}

The simplest functional forms for the force resisting the motion of a solid body with respect to some other medium were proposed by Stokes and Coulomb. Stokes found a drag force linear in the relative velocity $v$ between a solid and a fluid, while Coulomb suggested that the kinetic friction $F_{k}$ between two lubricated solids is independent of velocity. Later analysis $^{1}$ showed that solid friction tends to be weakly, i.e., logarithmically dependent on $v$, which will still be referred to as Coulomb friction in this work. Both laws are well understood from microscopic principles; ${ }^{2}$ a system close to thermal equilibrium will exhibit Stokes damping (unless it is close to a critical point), while the same system will show Coulomb friction when it is driven so quickly that instabilities are induced that push the system far away from thermal equilibrium. These two limiting cases can be best rationalized within the Prandtl-Tomlinson (PT) model, ${ }^{2,3}$ which is the most generic model exhibiting the logarithmic dependence of $F_{k}$ on $v$ known from Coulomb friction as well as the linear friction known from Stokes.

The PT model describes a point particle of mass $m$ that is dragged through a one-dimensional, sinusoidal potential $V(x)$ with a spring of stiffness $k$ moving at a velocity $v$. In the original interpretation, the point particle corresponded to an atom and the spring represented the elastic coupling of a surface atom to the equilibrium site in the host solid. It was also used to study the mean-field dynamics of driven charge-density waves. ${ }^{4}$ When applied to atomic-force-microscopy (AFM) experiments, ${ }^{5-12}$ the point particle is associated with the AFM tip and $k$ corresponds to the combined tip-cantilever stiffness. If $k$ is less than the maximum value of $-V^{\prime \prime}(x)$, the mass point will become unstable at given instances of time, which then, as described in much detail in the literature, leads to stick-slip motion and thus hysteresis, or in other words to significant friction. The predominant interest in the PT model during the last decade stems from the possibility to rationalize-if not to reproduce quantitatively-AFM experiments with it. If properly parameterized and/or generalized by a series coupling of two springs or by including higher-order harmonics to the substrate potential, it produces stick-slip curves in close similarity to those obtained with AFMs. ${ }^{5-13}$

The velocity dependence of the average (or kinetic) friction, as opposed to the average maximum friction during stick slip, has been evaluated surprisingly little in theoretical treatments of the PT model over more than two decades in velocity. In the few works covering two or more decades, see, e.g., Refs. 14 and 13, only overdamped dynamics were considered in the regime where Coulomb's friction law is applicable. Discussing kinetic friction over broad velocity ranges was yet central to Prandtl's original work. He proposed that one should distinguish between two regimes; in one, the spring moves so slowly that the point particle has enough time to jump back and forth across the barrier (i.e., it is in the linear response regime, in today's terminology) and in another, the spring moves so quickly that thermal fluctuations will make the atom cross the barrier once before the true instability is reached, at which point the old (meta)stable site disappears. In these two regimes, the following functional dependencies were postulated:

$$
F_{k}= \begin{cases}\gamma_{\mathrm{eq}}(T) v-\mathcal{O}\left(v^{3}\right), & \text { for small } v, \\ F_{\text {ref }}\left(T, v_{\text {ref }}\right)+a(T) \ln \left(v / v_{\text {ref }}\right), & \text { for large } v,\end{cases}
$$

see Eqs. (26) and (18) in the original work, ${ }^{2}$ which has recently been translated by Popov and Gray into English and will soon appear in press. ${ }^{15} \mathrm{In} \mathrm{Eq.} \mathrm{(1),} \gamma_{\mathrm{eq}}(T)$ is the equilibrium damping felt by the driving stage or spring. When instabilities are present, this (equilibrium or "effective") damping $\gamma_{\mathrm{eq}}$ much exceeds and is usually at most logarithmically dependent on the damping that is put explicitly into the interaction between mass point and substrate ${ }^{16}$ (see also model and result section). Unless the temperature is high, the logarithmic regime tends to span many decades, until $v$ becomes so large that inertial effects set in, or until the ad hoc damping dominates the merely logarithmically increasing friction. In this latter regime, $F_{k}$ starts to depend on many details (overdamped versus underdamped, explicit damping between mass point and substrate versus damping within the spring). For this reason, the regime of extremely large velocities shall not be of interest 
here. Moreover, it would be beyond $v=0.1$ in reduced units, which corresponds to difficult-to-reach $\approx 1 \mathrm{~mm} / \mathrm{s}$ in AFM experiments or $\approx 100 \mathrm{~m} / \mathrm{s}$ in atomistic interpretations of the PT model, at which point the starting assumptions of the PT model such as negligible local heating and the absence of wear would automatically break down anyway.

The remaining terms in Eq. (1) are a friction force $F_{\text {ref }}$ measured at a reference velocity $v_{\text {ref }}$, which can be chosen at will within the $\ln v$ regime, and a factor $a(T)$ stating how quickly $F_{k}$ changes with $\ln v$. In a first approximation, Prandtl found $a(T)$ to be linear in $T$, while in a more refined treatment, he suggested that $a(T)$ should scale with temperature according to

$$
a(T) \propto T^{2 / 3},
$$

see Eq. (23) in Prandtl's work. ${ }^{2}$

What Prandtl discussed only little is the question of how the small-velocity response crosses over to the large-velocity regime. This crossover, however, is of interest in a variety of fields. For example, in the mathematical description of tectonic plate motion, the singularities that occur for $\ln v$ in the limit of small $v$ need to be removed. ${ }^{17}$ This can done by modifying Eq. (1), e.g., through

$$
F_{k}=F_{0}+a(T) \ln \left(1+v / v_{\mathrm{c}}\right),
$$

where the new reference velocity $v_{\mathrm{c}}$ cannot be chosen arbitrarily anymore, because a change of $v_{c}$ can no longer be compensated through a different value of $F_{0}$. The velocity $v_{c}$ lies in between the low- and high-velocity regimes of friction. A disadvantage of this latter equation, at least when applied to the PT model, is that it does not reproduce linear response in the limit of small $v$, unless $F_{0}=0$. In that case, the equilibrium damping can be expressed as

$$
\gamma_{\mathrm{eq}}(T)=a(T) / v_{\mathrm{c}}(T) .
$$

Please note that not every contact must reach the linear response regime, for instance, when junction growth occurs more quickly than the thermally activated lateral motion ${ }^{16}$ so that choosing positive values for $F_{0}$ may sometimes be a better choice. ${ }^{17}$

Another disadvantage of Eq. (3) is that a Taylor series expansion of $F_{k}$ contains even powers of $v$. These, however, would be symmetry forbidden in any systematic expansion of $F_{k}(v)$, as it can be done within linear response theory, at least as long as the potentials used possess inversion symmetry. In this context, it is interesting to note that Prandtl's leading order correction to linear response was negative and of order $v^{3}$, see Eq. (1).

In this work, I attempt to find a friction-velocity relationship that possesses the proper symmetry and exhibits the correct linear and logarithmic limits at small and large velocities, respectively. This attempt will be complemented by numerical solutions of the appropriate Langevin equation for the PT model by molecular dynamics (MD) simulations. They allow one to vary temperature and velocity over a broad range of values without affecting the model parameters, something that might be difficult to achieve experimentally, and more importantly, one can produce reference data at absolute zero. Another aspect of this work is to discuss the relevance of the predicted $\left\{T \ln v / v_{0}\right\}^{2 / 3}$ dependence of friction. ${ }^{18,19}$ The underlying theories will be generalized to facilitate comparison to experiment or numerics.

The remainder of this article will be organized as follows: in Sec. II, I introduce the PT model, discuss the benefit of well chosen units so that kinetic friction, damping, and temperature can be expressed as meaningful dimensionless numbers, and include some typical values for these numbers. Section III contains details of the numerical implementation for the solution of the Langevin equation, which proved to be slightly more challenging than anticipated. In Sec. IV, I discuss various asymptotic limits for the friction velocity relationship and suggest an expansion for $F_{k}(v)$. Section V contains the results of the MD simulations. The last section contains, not surprisingly, discussions and conclusions. The rate theory for the PT model and some simple extensions are presented in Appendix A.

\section{MODEL}

Terms entering the PT model are the amplitude $V_{0}$ of the sinusoidal corrugation potential $V_{0} \cos (2 \pi x / L)$, the lattice constant $L$, the stiffness $k$ of the spring with which the point of mass $m$ is drawn, the "ad hoc" damping coefficient or inverse slip time $\gamma$, the driving velocity $v$, and the thermal energy $k_{B} T$. As usual, one should first define a unit system to describe the model so that a meaningful comparison between different experiments and simulations can be made. SI units are not really helpful, since stating that the velocity is, say $1 \mathrm{~cm} / \mathrm{s}$ leaves one clueless as to whether this should be considered extremely large or extremely small. The reason is that such an evaluation would depend on the remaining system parameters. Conversely, in well chosen units, a velocity of 0.01 can safely be assumed to be outside the extreme high-velocity regime (unless the damping were less than 0.01), albeit not necessarily small enough to be in the linear response regime.

Thoughtful theoretical studies ${ }^{4}$ tend to fix the units of energy and length by choosing $V_{0}=1$ and $2 \pi / L=1$. (This way all analytical formulas become compact, numerical solutions require only the minimum possible amount of floating point operations, and the static friction force automatically equals unity.) One can still define the unit of time. When considering the PT model in the overdamped limit, this can only be done through the damping coefficient. However, in the context of AFM tips and atomistic interpretations, it might be more sensible to fix the mass to $m=1$ and leave $\gamma$ as a system parameter. The rationale is that $m$ would not change significantly between different experiments, while $\gamma$ can change with load - and more importantly $-\gamma$ is an interfacial property rather than a property of the tip or tip/cantilever system, and thus much more difficult to gauge. Moreover, the choice of $m$ as the unit of mass allows one to find more easily estimates for how to translate typical AFM scanning speeds into reduced units.

With the current choice of units, the equation of motion for the PT model under white thermal noise then reads

$$
\ddot{x}+\gamma \dot{x}+k(x-v t)=\sin x+\Gamma(t),
$$

where $v$ is the sliding velocity of the tip and $\Gamma(t)$ the white random force satisfying the fluctuation dissipation theorem, i.e., $\left\langle\Gamma(t) \Gamma\left(t^{\prime}\right)\right\rangle=2 \gamma k_{B} T \delta\left(t-t^{\prime}\right)$. From Eq. (5), one can 
learn that four independent, dimensionless variables remain: (i) $k$, (ii) $\gamma$, (iii) $v$, and (iv) $k_{B} T$, which are worth discussing separately.

(i) When $k$ is greater than unity, no instability can occur, 2,6 which would be of little interest for our study. When $k$ is much less than one, there are always many different metastable sites, which can lead to nonmonotonic behavior of the friction force on velocity, in particular for underdamped systems. When $k$ is less but not much less than one, the number of sites where the mass point is (meta)stable is one or two depending on the position of the spring. The motion of the mass point when pulled under suitable conditions is then a stick-slip motion with the period of the underlying lattice. This is the condition encountered in the majority of AFM experiments and motivates the default choice of $k=0.4$. I will mention explicitly whenever $k$ differs from that value. Note that some experimentalists use $\eta=1 / k$ as the dimensionless control parameter $^{7}$ and find values in the vicinity of $\eta=2$, which translates to $k=0.5$.

(ii) A value of $\gamma \approx 1$ subdivides the motion of the mass point between underdamped and overdamped. This ad hoc damping, which can be motivated and derived from quite rigorous linear response treatments, reflects the direct coupling of the point particle to phonons and potentially electronic degrees of freedom of the substrate. ${ }^{16,20,21}$ Most theories focus on the overdamped limit (e.g., by choosing the unit of time to be $1 / \gamma$ and then sending $m$ to 0 ), partly because this facilitates the solution of analytical theories. Conversely, AFM tips do not seem to be overdamped. Otherwise, multiple slips observed in AFM experiments using high loads, resulting in small $k$, would not be observed. ${ }^{22}$ For this reason, I will chose a value for damping that represents the underdamped case, namely $\gamma=0.1$, However, I will demonstrate explicitly in one case, which should reflect room-temperature AFM experiments reasonably well, that $\gamma$ affects $F_{k}(T, v)$ at most logarithmically as long as $v$ is not extremely large.

Typical values of the ad hoc damping for AFM tips when almost in contact in the substrate ${ }^{21}$ are close to $10^{-12} \mathrm{~kg} / \mathrm{s}$, which would lead to friction forces of $10^{-18} \mathrm{~N}$ at AFM velocities of $1 \mu \mathrm{m} / \mathrm{s}$. The resulting direct damping forces would thus be approximately nine decades smaller than the usually observed friction forces. Of course, this gap decreases when the tip is pressed harder against the substrate, and it may even happen that the tip motion becomes slightly overdamped. But even then would damping only matter at velocities approaching unity in dimensionless units.

(iii) $v$ and (iv) $k_{B} T$ are varied over a broad parameter range. Both numbers are usually small in regular AFM experiments. The advantage of the simulation is that $T$ can be raised sufficiently high so that the crossover from logarithmic-like and friction linear in velocity can be simulated directly quite easily. At the same time, MD simulations of the PT model can reach velocities smaller than those achievable experimentally. This claim certainly does not hold for explicit atom simulations.

Typical numbers for AFM experiments are (static) friction forces on the order of a few nano Newtons, say $10 \mathrm{nN}$, while the distance between two adjacent metastable sites is around $3 \AA$. This makes the unit of energy be roughly $3 \times 10^{-18} / 2 \pi \mathrm{J}$, which in turn moves room temperature $k_{B} T$ into the vicinity of $10^{-2} V_{0}$. It is difficult to determine accurate numbers for the mass $m$, in particular as it seems necessary to use a series coupling of two springs to describe dynamics properly. ${ }^{10}$ In order to come up with some number, I shall assume that the value of $m_{\text {tip }} \approx 10^{-12} \mathrm{~kg}$ as used in Ref. 7 was nevertheless a reasonable choice. This makes the unit of velocity become

$$
\begin{aligned}
{[v] } & =\sqrt{\frac{10 \mathrm{nN}(3 \AA / 2 \pi)}{10^{-12} \mathrm{~kg}}} \\
& \approx 7 \times 10^{-4} \mathrm{~m} / \mathrm{s},
\end{aligned}
$$

so that the "natural" unit of velocity for AFM tips is in the order of $\mathrm{mm} / \mathrm{s}$, although this value may vary with load by a decade. Given the current number, one can argue that the range of velocities from $10^{-7}$ to $10^{-1}$ analyzed in the result section would then cover a range from $1 \AA / \mathrm{s}$ through $0.1 \mathrm{~mm} / \mathrm{s}$. If, however, we compared the same calculations to the originally intended atomistic interpretation of the PT model, then the same dimensionless numbers should be interpreted as $10^{-7}$ to $10^{-1}$ times the speed of sound $c_{s}$. The investigated velocity range would then correspond to $0.1 \mathrm{~mm} / \mathrm{s}$ through $100 \mathrm{~m} / \mathrm{s}$, assuming that $c_{s}$ is on the order of $1000 \mathrm{~m} / \mathrm{s}$. Thus, depending on the context, the velocity of $1 \mathrm{~cm} / \mathrm{s}$ turns out very large in reduced units for AFMs, approximately ten, and utterly small, $\approx 10^{-5}$, when comparison is made to atomistic dynamics.

Given the above considerations on the AFM, the unit of time $[t]$ becomes $\approx 0.1 \mu \mathrm{s}$. A damping coefficient in the order of unity in reduced units would thus damp out the oscillatory motion of a tip after a stick-slip event during this time. Since this is hardly ever the case, one can safely assume that $\gamma$ should almost always be less than unity in wearless friction experiments.

The meaning of the thermal energy in reduced units also needs to be addressed. As stated in a preceding paragraph, room-temperature thermal energy would be $\approx 0.01$ in reduced units. However, this does not necessarily imply that temperature is small. As $k$ approaches unity, the relevant barrier is not twice the corrugation potential $V_{0}$ but it is the barrier $\Delta E_{\mathrm{b}}$ that arises from the combined substrate-atom and spring-atom potential when there are two degenerate mechanical equilibrium sites. For our default value of $k=0.4$, $\Delta E_{\mathrm{b}}$ turns out to be $\approx 0.6 V_{0}$, so that thermal energy would have to be associated with $k_{B} T \approx 0.01$. The relevant barrier can decrease drastically and thus the ratio $k_{B} T / \Delta E_{\mathrm{b}}$ can become very large when the load on the tip is reduced as done in Refs. 6 and 23. AFM experiments should therefore be able to probe the transition from linear to logarithmic friction too, although difficulties associated with proper sampling, discussed in the model section, are likely to impede such an analysis quite substantially, even if the resolution of lateral forces were as high as in surface force apparatus experiments.

In the remaining part of this section, the athermal limit of the $k=0.4$ default PT model will be discussed. As it should always be done when analyzing a PT model, be it the original one or a generalization of it, the energy barrier $\Delta E_{\mathrm{b}}$ mentioned earlier should be ascertained as well as the instability energy $\Delta E_{\mathrm{i}}$. The latter is the energy that is released nonadiabatically when a slowly driven athermal particle becomes unstable. Both energies can be easily obtained to a desired numerical accuracy 


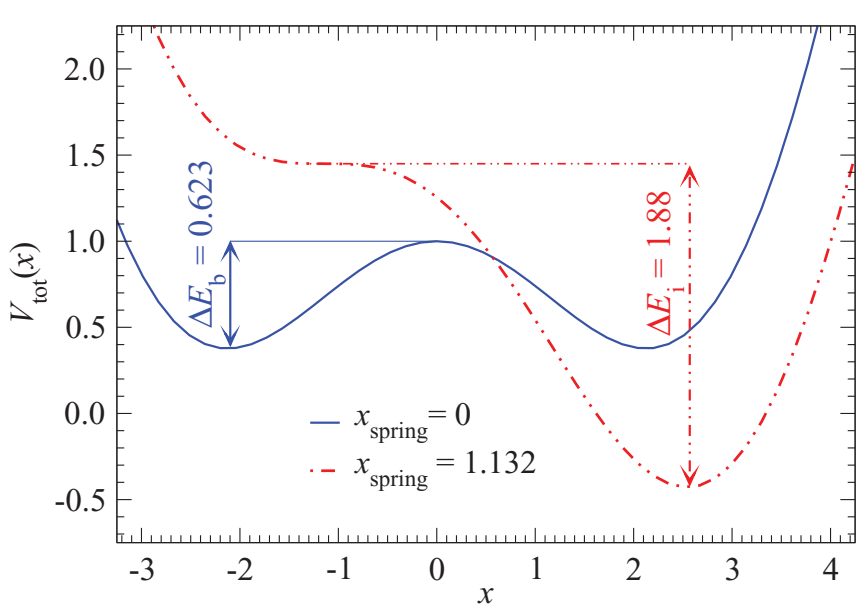

FIG. 1. (Color online) Combined substrate and spring potential $V_{\text {tot }}$ for two selected positions of the $k=0.4$ spring as a function of position $x$. For $x_{\text {spring }}=2 n \pi$, the two mechanically stable sites are degenerate, which allows one to read off the activation barrier $\Delta E_{\mathrm{b}}=$ $0.6231(5)$. Near $x_{\text {spring }}=2 n \pi+1.132$, the left minimum disappears, which leads to an instability energy of $\Delta E_{i}=1.87(8)$, from which the athermal, low-velocity limit kinetic friction force $F_{k}=\Delta E_{i} / 2 \pi=$ 0.298(9) follows.

by plotting the total energy as a function of the position $x$ of the point particle, for different spring positions. This is done in Fig. 1. There are also analytical solutions, which will be discussed in Appendix A.

From $\Delta E_{\mathrm{b}}=0.62$, one can conclude that it will be impossible to find a logarithmic $F_{k}(v)$ dependence unless thermal energies are distinctly less than 0.62. Otherwise, the likelihood of a particle to sit at the top of the barrier is (almost) just as high as to sit in one of the minima, in particular as the curvature near the barrier is small. From $\Delta E_{\mathrm{i}}$ one can ascertain that the athermal, low-velocity limit kinetic friction will be $F_{k}=\Delta E_{i} / 2 \pi=0.298(9)$. This is because the particle is going to lose this energy irreversibly as heat, each time the spring advances by one lattice constant, which is $2 \pi$ in reduced units. The kinetic friction as obtained by MD (see next section) is shown in Fig. 2.

For small $v$, the $F_{\mathrm{k}}(T=0, v)$ dependence can be well approximated by the relation ${ }^{4}$

$$
F_{k}=F_{k, \text { ideal }}+\text { const } v^{2 / 3},
$$

where the constant depends on $k, \gamma$, and $m$ but not on velocity. At large $v$, this relation breaks down, when the point particle can no longer dissipate the energy that was meant to get lost as heat from previous instabilities when it encounters the next instability point, e.g., when $\gamma v$ approaches unity. Specifically, the point particle still has kinetic energy before an instability point is reached so that it is crossed prematurely. For other parametrizations of the PT model, there can be other reasons for the breakdown of Eq. (8) at high spring velocities. However, the only point that matters for the subsequent analyses is that the extremely large velocity range starts at $v \approx 0.1$ for our model and that the suggested finite-temperature treatments cannot be used for that regime.

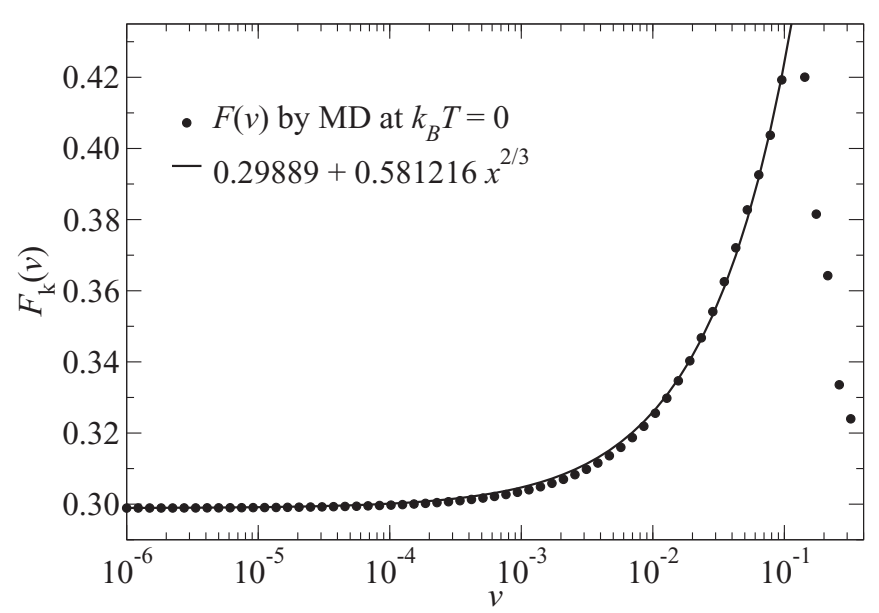

FIG. 2. Kinetic friction $F_{k}$ as a function of velocity $v$ for $k=$ 0.4 and $\gamma=0.1$. The low-velocity law $F_{k}=F_{k \text {,ideal }}+$ const $v^{2 / 3}$ is accurate up to a velocity of $v=0.1$, where inertial effects are starting to play a role. The value for $F_{k \text {,ideal }}$ is equal to that obtained from the analysis of the instability energy in Fig. 1.

\section{METHODS}

The velocity Verlet algorithm was used to integrate the equations of motion in the molecular dynamics simulations. In this scheme, damping and random forces were treated on equal footing with the conservative forces, which-at least for the small values for the damping term $\gamma$ used in this study-gives faster convergence of thermodynamic averages (such as internal energy when the spring is at rest) than if they had been excluded from the corrector step of the velocity Verlet scheme. The time step $d t$ was chosen much smaller than any intrinsic time scale inherent to the system: $d t=1 /\{40(\sqrt{1+k}+\gamma)\}$. (Remember that $1+k$ is the maximum curvature of the combined spring-substrate potential in the given unit system.) The random forces were uniformly distributed and deduced from the xorshift random number generator (XOR), ${ }^{24}$ through $\Gamma=\Gamma_{0}\left(2 u_{\text {xor }}-1\right)$. The first moment of $\Gamma$ disappears and $\Gamma_{0}$ was chosen so that it satisfied the fluctuation dissipation theorem, i.e., $\Gamma_{0}=$ $\sqrt{6 \gamma k_{B} T / d t}$. Owing to the law of large numbers in the limit $d t \rightarrow 0$, it suffices to produce the correct first and second moment of the random force so that there is no need for the generation of Gaussian random numbers. This makes it unnecessary to take sine, cosine, and logarithms of random numbers within the Box Muller algorithm. These would have been expensive operations with additional potential "dangers" when the uniform random numbers entering the Box-Muller algorithm are too close to zero or too close to one.

Surprisingly, the $\mathrm{r} 250$ random number generator, which was used in the first few production runs, frequently induced drifts in the ratio of $F(v) / v$ at very small $v$, i.e., the quotient first went onto a plateau indicative of Stokes friction, but then either decreased or increased quite substantially when $v$ was further reduced. This drift could not be related to a drift in the $\mathrm{r} 250$ itself. Even with the XOR shift random number generator, one needs to be cautious and discard the first few million random numbers in order to ensure that the algorithm has run in. Without doing so, one risks to inject too much energy into 
the system during those first few steps, supposedly due to some strong correlations that tend to occur during the warmup phase.

In the present study, the sliding velocity was varied over more than six decades for small $T$, i.e., for $k_{B} T \ll \Delta E_{\mathrm{b}}$, at a maximum statistical noise in $F_{k}$ of $5 \%$. Here, $\Delta E_{\mathrm{b}}$ denotes the energy barrier between the two (degenerate) stable sites when the pulling spring is located on a maximum of the corrugation potential. At large temperatures, only three decades in sliding velocity could be covered. The reason why it is difficult to cover more decades at large $T$ is that the linear response regime at high $T$ is reached already at moderate $v$. In this regime, the signal and thus the signal to noise ratio goes down by a factor of two, when the velocity is halved. Therefore one needs to slide four times the distance in the low-velocity simulation as compared to the simulation at the original velocity, in order to compute $F_{k}$ with a fixed (relative) stochastic error. An additional factor of two arises due to the need for performing twice as many MD time steps to slide the mass point at half the velocity. Thus, dividing the velocity by two in the linear response regime makes the number of required floating point operations go up by a factor of eight. This implies that adding a decade at small velocities cranks up the compute time by a factor of thousand. To access one or two more decades, one should therefore port these (low-memory) calculations to graphical processing units.

The large number of time steps at small velocity and the poor signal-to-noise ratio induces numerical problems. One can no longer simply add up the instantaneous forces and then divide by the number of observations. An extreme case would be that the noise is on the order of unity and the signal is in the order of $10^{-4}$. If all observations were stochastically independent (which they are not when the mass point needs to sample different valleys), one would need $10^{8}$ independent (!) observations to get an average with an error bar of $100 \%$ and thus $10^{12}$ observations to reduce the error bar to $10 \%$. In a naive approach, one would therefore lose 16 relevant digits, which is roughly the accuracy of a long double precision variable in $\mathrm{C}++$. It was therefore assured that subtotals were conducted so that never more than two numbers were added up onto one counter, and the result of two summands was passed up to the next hierarchy.

Fitting the $F_{k}(v)$ data to different functional forms did not prove to be trivial either, because the fits should be sensitive to $F_{k}(v)$ at large $v$ (low temperature) and to the effective damping $\gamma_{\text {eff }}(v)=F_{k}(v) / v$ at small $v$ (high temperature). Due to these two constraints, the function $f(v)=F_{k}(v) / \sqrt{v}$ was considered, which is sensitive to the velocity regime where the response crosses over from linear to logarithmic. The reason is that $f(v)$ first increases with $v$ in the linear response regime and then decreases when $F_{k}(v)$ becomes logarithmic in $v$. Whenever the maximum of $f(v)$ was inside the observation window, the $\chi^{2}$ fits were based on $f(v)$. Whenever $f(v)$ increased or decreased monotonically within the observation window, $\chi^{2}$ calculations were based on $\gamma_{\mathrm{eff}}(v)$ and $F_{k}(v)$, respectively.

\section{ASYMPTOTIC ANALYSIS}

In the introduction, it was argued that the function $F_{k}(v)$ in Eq. (3) contains the proper small- and high-velocity limits for
$F_{0}=0$, however, it contains symmetry-forbidden even-power terms in $v$. The inverse hyperbolic sine function $\operatorname{arsinh}(x)$ behaves like $\ln (1+x)$ but only contains odd powers in $x$. It is linear in $x$ at small $x$ with the same prefactor as $\ln (1+x)$, while for large $x, \operatorname{arsinh}(x)$ approaches $\ln (1+x)$ asymptotically. The two functions never differ more than by roughly $30 \%$, although their inverse functions differ by a factor of two when their arguments become large. This is a small deviation given that the most serious attempts to date to connect AFM experiments and all-atom simulations still miss a little more than a decade in sliding velocity when trying to match experiments and simulations. ${ }^{25}$ In fact, the maximum $30 \%$ difference between $\ln (1+v)$ and $\operatorname{arsinh}(v)$ may be too small for us to discriminate clearly between the two alternatives when fitting the two functions to experimental or numerical data: The "fit" parameters $a(T)$ and $v_{\mathrm{c}}(T)$ will turn out slightly different in each approach so that six decades in velocity and numerical uncertainties in $F_{k}(v)$ on the order of $5 \%$ are insufficient to clearly reveal that the arsinh approach is superior to the better known logarithmic dependence.

According to linear response theory, the response of any finite-size and finite-temperature system in which particles interact through well-behaved potentials is an analytical function in the driving force and vice versa. It should thus be possible to expand $F(v)$ into powers of $v$, where only odd powers are allowed for potentials with inversion symmetry. A simple Taylor series expansion, however, will necessitate many terms to reflect the logarithmic $F_{k}(v)$ dependence at large $v$. It will thus be more efficient to use kernels that automatically ensure that each term does not grow too quickly when $v$ becomes large. Such an expansion would be

$$
F_{k}(T, v)=\sum_{n} a_{n}(T)\left\{\operatorname{arsinh}\left[v / v_{\mathrm{c}}(T)\right]\right\}^{n},
$$

where $n$ only runs over odd integers when the potentials possess inversion symmetry. The relation to the original Taylor series expansion can be achieved as usual by expanding the kernel function into powers of $v$ and then by successively matching the expansion coefficients; the first $N$ orders in the Taylor series will fix $v_{\text {c }}$ plus the $N-1$ leading $a_{n}$ coefficients. As it will turn out later, one can usually truncate after the $a_{3}$ term, unless the temperature is so small that the response starts to resemble that of the athermal PT model. In that case, it is easier to add a $v^{2 / 3}$-type correction to the leading-order $a_{1}$ term rather than to add more higher-order $a_{n}$ terms.

What has not yet been discussed in detail in this work is how $a(T)$ and $v_{c}(T)$ are expected to depend on temperature. To investigate this issue, it is instructive to analyze how we expect $F_{k}(T, v)$ to behave in the limit of small velocities and in the limit of small temperatures. Interestingly, there is no unique value for $F_{k}(T \rightarrow 0, v \rightarrow 0) .{ }^{26}$ As long as $T$ is finite, there will always be a linear response term at small $v$, and thus

$$
\begin{aligned}
F_{k}(0<T, v \rightarrow 0) & =\lim _{v \rightarrow 0} \gamma_{\mathrm{eq}}(T) v \\
& =0 .
\end{aligned}
$$

Conversely, if we assume that $v$ is small but finite and $k$ is less and not too close to one, then we can exploit the results for the 
athermal Prandtl-Tomlinson model:

$$
F_{k}(T \rightarrow 0,0<v \ll 1)=F_{k, \text { ideal }}+\mathcal{O}\left(v^{2 / 3}\right) .
$$

By comparing Eqs. (10) and (12) one can see that $F_{k}(0,0)$ disappears when we first send velocity to zero and then

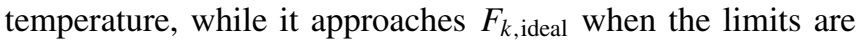
carried out the other way around. Any functional dependence of $a(T)$ and $v_{\mathrm{c}}(T)$ should reflect this behavior.

Suggestions for the functional dependence for $a(T)$ have been $a(T) \propto T$ and $a(T) \propto T^{2 / 3}$, which can be written as

$$
\frac{a(T)}{a_{\text {ref }}}=\left(\frac{T}{T_{\text {ref }}}\right)^{\alpha},
$$

where $\alpha$ is supposed to be either unity or two thirds and $T_{\text {ref }}$ is an arbitrary reference temperature. At small temperature, the functional dependence for $\gamma_{\mathrm{eq}}(T)$ can be assumed to follow an Arrhenius dependence so that

$$
\frac{\gamma_{\text {eq }}(T)}{\gamma_{\text {ref }}}=\frac{T_{\text {ref }}}{T} \exp \left[\beta \Delta \mathcal{F}_{\mathrm{b}}(T)-\beta_{\text {ref }} \Delta \mathcal{F}_{\mathrm{b}}\left(T_{\text {ref }}\right)\right],
$$

where $\Delta \mathcal{F}_{\mathrm{b}}(T)$ is a potentially temperature-dependent free energy barrier and $\beta$, as usual, the inverse thermal energy $1 / k_{B} T$.

We will now be concerned with the following question: if one were to extend Eq. (14) to small $T$ and assume that the leading-order term in the arsinh expansion were dominant even at large $v$, what would the functional dependence of $\Delta \mathcal{F}_{\mathrm{b}}(T)$ have to be, so that we recuperate the correct value for $F_{k}$ when we first send $T$ to zero and then $v$ ? To address this question, one can write the first term in the arsinh series as

$$
F_{k}(T, v) \approx a(T) \operatorname{arsinh}\left[1+\gamma_{\mathrm{eq}}(T) v / a(T)\right],
$$

which in a first approximation would become

$$
\lim _{T \rightarrow 0} F_{k}(T, v) \approx \frac{a(T) \Delta \mathcal{F}_{\mathrm{b}}(T)}{k_{B} T} .
$$

Given that this should be close to the zero-velocity, athermal $F_{k}$, it follows that

$$
\Delta \mathcal{F}_{\mathrm{b}}(T) \approx F_{k} \frac{k_{B} T}{a(T)} .
$$

This result appears meaningful if $a(T)$ is linear in $k_{B} T$ because the barrier $\Delta \mathcal{F}_{\mathrm{b}}$ would then converge to a constant at $T \rightarrow 0$. For the sublinear dependence, $a(T) \propto T^{2 / 3}$ one would have to conclude that the leading-order term in the arsinh series is not sufficient to describe the large velocity response, because a vanishing barrier is unphysical. Yet, at temperatures sufficiently high for the first term in the arsinh series to be the leading-order term, one can express $F_{k}(T, v)$ as a function of $T$ and $F_{k}$ as well as of the two parameters $a_{\text {ref }}$ and $\gamma_{\text {eq }}\left(T_{\text {ref }}\right)$ that need to be gauged at a given reference temperature.

\section{RESULTS}

\section{A. Functional form of $F_{k}(v)$}

In this section, we will first be concerned with the question if the expansion in inverse hyperbolic sine functions is appropriate. For this purpose, MD results will be compared to $\operatorname{an} \operatorname{arsinh}\left(v / v_{\mathrm{c}}\right)$ expansion and to another expansion, in

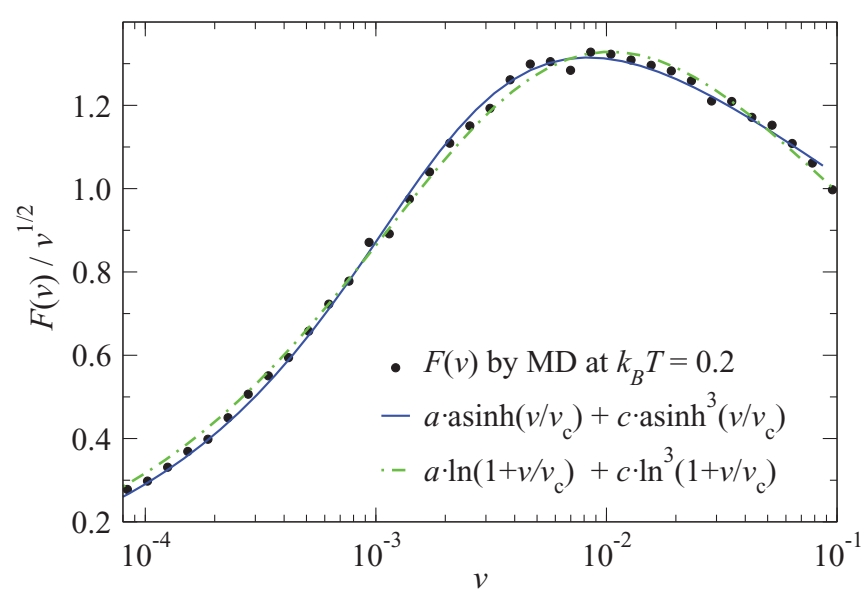

FIG. 3. (Color online) $F_{k} / \sqrt{v}$ as a function of velocity $v$ for the default model at $T=0.2$. The MD data are fit to two different model functions.

which the kernel function is replaced with $\ln \left(1+v / v_{\mathrm{c}}\right)$. This comparison, in which the target function was $F_{k}(v) / \sqrt{v}$ is presented in Fig. 3.

As explained in the last section, the small difference between the functions $\operatorname{arsinh}(x)$ and $\ln (1+x)$ makes it difficult to ascertain if an $\operatorname{arsinh}\left(v / v_{\mathrm{c}}\right)$ expansion really is superior to one based on $\ln \left(1+v / v_{\mathrm{c}}\right)$. As a matter of fact, the $\chi^{2}$ deviations tend to be very similar to each other between the two approaches. However, an important clue that only odd powers in $v$ should occur comes from the observation that including $\operatorname{arsinh}\left(v / v_{\mathrm{c}}\right)$ or $\ln \left(1+v / v_{\mathrm{c}}\right)$ terms raised to the third power decreases the $\chi^{2}$ error distinctly more than if the respective second-power terms are used. What is nevertheless somewhat distressing is that the values for $a_{1}(T)$ and $v_{c}(T)$ differ surprisingly much, specifically, the fits yielded $v_{c}(\operatorname{arsinh})=\exp (-6.47), a_{1}(\operatorname{arsinh})=0.0450$, and $a_{3}(\operatorname{arsinh})=0.000936$, as opposed to $v_{c}(\ln )=\exp (-5.95)$, $a_{1}(\ln )=0.0838$, and $a_{3}(\ln )=0.0001096$, i.e., the estimates for the term $a\left(k_{B} T=0.2\right)$ differ almost by a factor of two. This might have been expected, as the thermal energy is not much less than the energy barrier $\Delta E_{b}$, and the curves do not show much of a logarithmic dependence to begin with, as the domain right of the maximum of $F_{k} / \sqrt{v}$ barely covers a decade. In contrast, the deduced equilibrium damping terms come out within $10 \%$ of one another, that is, $\gamma_{\mathrm{eq}}(\operatorname{arsinh})=29.1$ versus $\gamma_{\mathrm{eq}}(\ln )=32.3$.

As temperature decreases, the higher-order correction terms can be replaced with the $v^{2 / 3}$-corrections known from the $F_{k}(T=0, v)$. This is demonstrated in Fig. 4, where the thermal energy is $k_{B} T=0.1$.

A danger one has to be aware of when adding the athermal $v^{2 / 3}$-correction from the athermal PT model is that it decays with a power smaller than unity. This correction must therefore be damped off at small $v$, for example, with a factor $\left[1-\exp \left(-v / v_{c}\right)\right]$, in order to ensure the proper linear response. An introduction of such a correction factor for the velocity range shown in Fig. 4 was not needed to obtain the satisfactory agreement between theory and simulation. That situation would have started to change if more decades had been added at small velocities. 


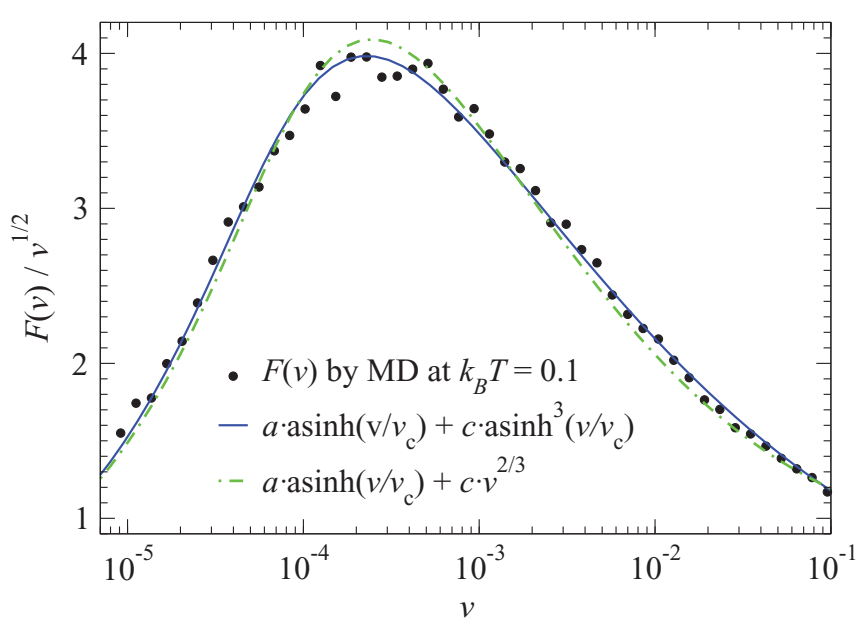

FIG. 4. (Color online) $F_{k} / \sqrt{v}$ as a function of velocity $v$ for the default model at $T=0.1$. The MD data are fit to two different model functions. The prefactor to the $v^{2 / 3}$ correction was not a fit parameter but identical to the value identified in Fig. 2.

For $T=0.1$, the two competing fits now give values consistent within $10 \%$ for both $a_{1}$ and $\gamma_{\text {eq }}$, namely $a_{1}=0.0274$ and $\gamma_{\mathrm{eq}}=485$ for the regular third-order arsinh correction versus $a_{1}=0.0317$ and $\gamma_{\mathrm{eq}}=445$ when the power-law correction is applied.

One might be concerned that the excellent agreement between the (low-order) arsinh expansion and numerical data is fortuitous, because of the particular value of $k$. In order to investigate if this concern is true, additional simulations were run at $T=0.133$ for $k=0.3\left(F_{k}=0.20146\right)$ and $k=0.5$ $\left(F_{k}=0.42065\right)$. The choice for $T=0.133$ was motivated by the observation that the maximum of $F(v) / \sqrt{v}$, i.e., the crossover point between Stokes and Coulomb friction, was close to the center of the accessible window on a logarithmic velocity scale. For all curves, the quality of the fits was as good as the ones shown so far. The ratios of the arsinh coefficient $r=a_{3} / a_{1}$ turned out to be less than 0.03 in all three cases, specifically: $r(k=0.3) \approx 0.0074, r(k=0.4) \approx 0.0126$, and $r(k=0.5) \approx 0.0236$. Of course, in the limit $k \rightarrow 1^{-}$, the expansion becomes meaningless, because instabilities are no longer significant.

\section{B. Low-temperature results}

Analyses, as the ones carried out for $T=0.1$ and $T=0.2$, were repeated for a wide range of temperatures and velocities. Results from a representative subset are shown in the regular $F_{k}(v)$ representation in Fig. 5. The fits to the MD results clearly reveal that the first term in the expansion of Eq. (9) plus a small correction term describe the $F(v)$ behavior over several decades in temperature and sliding velocity.

All data sets were analyzed such that the power-law correction and the third-order arsinh term were assumed as the only correction to the $F(k)=a \operatorname{arsinh}\left(v / v_{\mathrm{c}}\right)$ leading-order term. While the power-law correction gave the slightly better fits (and were used for later analyses), it is yet useful to also investigate the prefactors $a_{3}$ for the third-order arsinh term. For $k=0.4$, they turned out to be, at most, close to $2 \%$ of the leading-order coefficient. This means that the

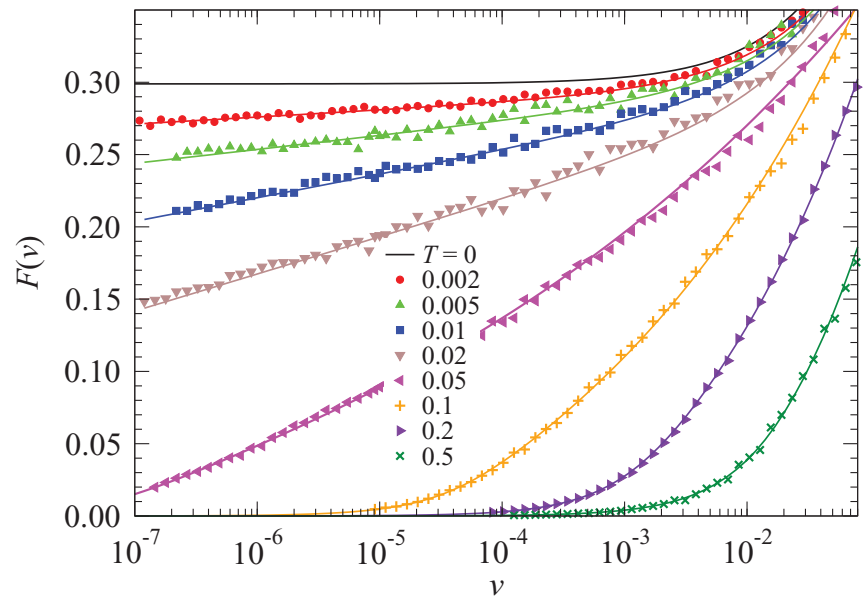

FIG. 5. (Color online) $F_{k}(v)$ as a function of velocity $v$ for the default model at temperatures covering more than two decades in magnitude. Lines are fits according to the same equations used in Fig. 4, where the third-order correction term corresponded to the power law at $T<0.1$, while the third-order arsinh term was used otherwise.

linear response behavior crosses over into a predominantly logarithmic velocity dependence at $v=v_{\mathrm{c}}$.

From the fits to the MD data, the rate at which kinetic friction increases with $\ln v$ or with $\operatorname{arsinh}\left(v / v_{\mathrm{c}}\right)$ can be ascertained. This allows one to test the scaling hypotheses for $a(T)$ with temperature. Results for the coefficient $a_{1}(T)$, or simply $a(T)$, are presented in Fig. 6. It clearly confirms the prediction that the leading-order prefactor in the arsinh expansion scales with $T^{2 / 3}$, although there appears to be no need to raise the $\operatorname{arsinh}(v)$ into a power different than one. However, confidence into the rate theory can be gained from the observation that the prefactor to the $a(T) \propto T^{2 / 3}$ relation matches almost perfectly the one derived from a slightly modified rate theory, which is presented in Appendix A.

The observed linearity of $F_{k}$ with $\operatorname{arsinh}(v)$ or $\ln (v)$ challenges recently presented rate theories in which it was

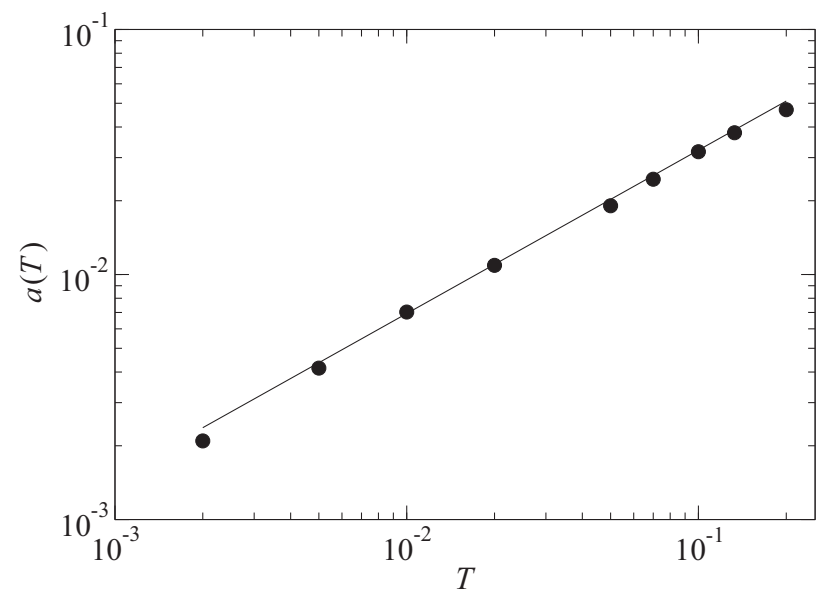

FIG. 6. Leading-order prefactor $a(T)$ as a function of $T$. The line, $0.149 T^{2 / 3}$, is a parameter-free result from a slightly modified rate theory in which the motion of the equilibrium site to which a depinning particle is jumping, was included. 


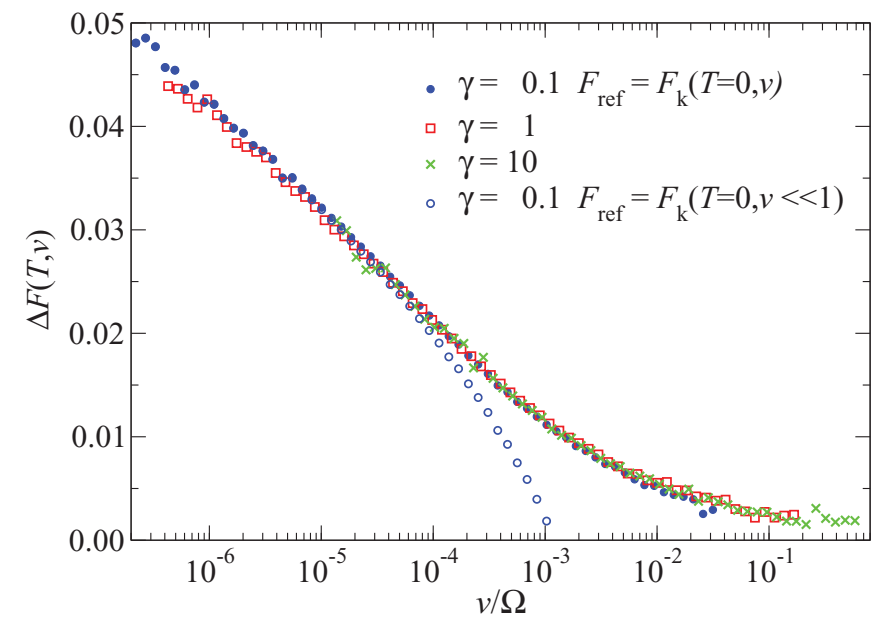

FIG. 7. (Color online) Difference between measured friction and reference friction $\Delta F(T, v)=F_{\text {ref }}\left(T_{\text {ref }}, v_{\text {ref }}\right)-F(T, v)$ as a function of velocity. Damping was varied from overdamped to underdamped. Velocity is divided by the eigenfrequency of the pulling spring, which made it possible to collapse results for different dampings. In one case (open circles), the reference friction was simply the ideal athermal kinetic friction.

found that $F_{k}$ changes with $\left[\ln \left(v / v_{0}\right)\right]^{2 / 3}$ and it solidifies previous theories. The generic ingredients to those rate theories, ${ }^{18,19}$ including some minor extensions, are presented in Appendix A. It has to be kept in mind that the rate theories had not been developed around the crossover from linear response to logarithmic friction but rather at a range of velocities that sit to the right of the $F(v) / \sqrt{v}$ maximum, but that do not enter yet the extremely high-velocity range. Specifically, the recent rate theories were concerned with the question of how the average distance from the average thermal depinning point to the athermal instability point depends on temperature when this depinning still occurs in the vicinity of the athermal instability point.

In order to give a more substantiated evaluation of the rate theories, one should compare the deviations between thermal and athermal kinetic friction well above $v_{\mathrm{c}}$, because this difference is what the rate theories are concerned with. To make the comparison between the computed $F_{k}$ and the rate theories clean, one needs to consider the motion of the equilibrium site, to which a depinning particle pops. These dynamics increase the thermal reduction of $F_{k}$ with respect to the one of the depinning force $F_{s}$ (which is the predominant quantity of interest in the rate theories), but the scaling remains invariant within the rate theory approach, which is shown in Appendix A.

Figure 7 shows the deviation between "measured" friction $F_{k}(T=0.005, v)$ at a small but finite temperature and the zero-temperature reference friction at $F_{k}(T=0, v)$. In order to make sure that damping did not affect the results in a qualitative fashion, different damping constants were considered.

It remains difficult to find support for the $\left[\ln \left(v / v_{0}\right)\right]$ behavior even in the new representation. The only case where one could fit more easily a $\left[\ln \left(v / v_{0}\right)\right]^{2 / 3}$-type function than the regular $\ln \left(v / v_{0}\right)$ term requires one to subtract a constant from the data, rather than the athermal $F_{k}(v)$ curve. The latter comparison, however, is the more meaningful one, because it

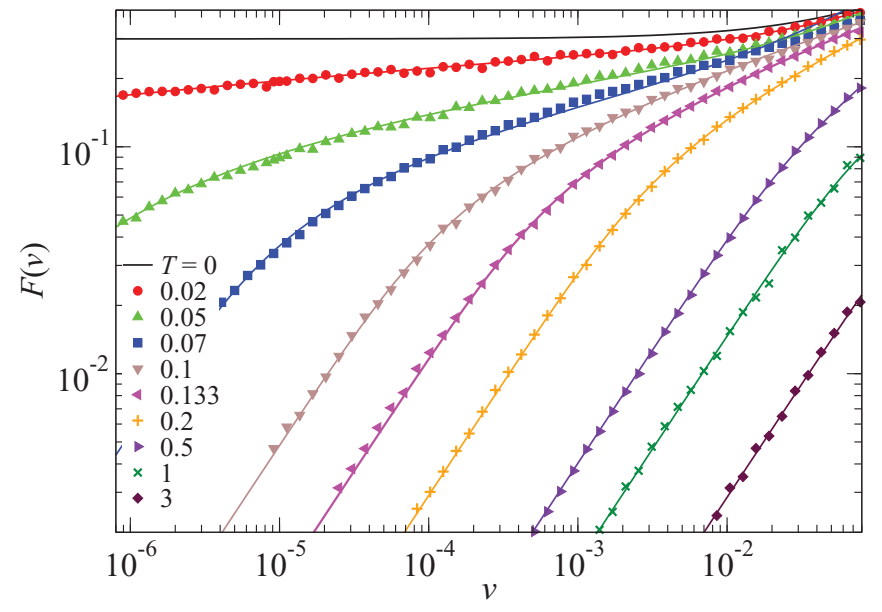

FIG. 8. (Color online) $F_{k}(v)$ as a function of velocity $v$ for the default model at temperature covering more than two decades in magnitude.

does not contain the athermal $v^{2 / 3}$ contribution from Eq. (8), which is not part of the rate theory for $\Delta F_{k}$. Since all steps of the rate theory are yet well justified, one may wonder why it is so difficult to reproduce the law with solutions of the Langevin equation. One reason may be that it must break down at large velocities, when $v$ approaches $v_{0}$. At that point a function that is right-curved crosses over into one that is left curved. For a power law that differs so little from an exponent of one, it may thus be necessary to cover many more decades (and to decrease temperature even further). This aspect is discussed further in Appendix B.

\section{High-temperature results}

At high temperatures, the friction law becomes Stokes-like, so that it is small at small velocities. It is then difficult to assess the validity of the arsinh expansion when the simulation data are represented linearly as is done in Fig. 5. To better reveal the effective damping, $F(v) / v$, more MD data is shown than before but this time in a double logarithmic representation, see Fig. 8. As before, it is possible to capture the simulation data quite reasonably with the fit function.

One can notice that some curves have higher scatter than others, which may be worth commenting on. It is simply a consequence of runs having been submitted with different requirements on the stochastic error. In each set of runs, the velocity is lowered in discrete steps, where the new velocity is a fraction (slightly larger than $80 \%$ ) of the previous velocity. At each velocity, subaverages of the spring force are performed that correspond to the average of the spring force over one lattice constant. These subaverages enable one to have $N_{n}$ independent estimates for $F_{k}$ from where a variance and an error bar can be computed. For the next velocity, one then assumes that the error bar will not change much and requests a number $N_{n+1}$ so that the expected statistical error at velocity $v_{n+1}$ remains below a set relative error. One has to avoid to adjust $N_{n}$ on the fly at velocity $v_{n}$, because this will induce an offset in the results (for reasons similar to why the maximum displacement in Monte Carlo simulations may not be varied during the observation in order to keep the acceptance ratio 


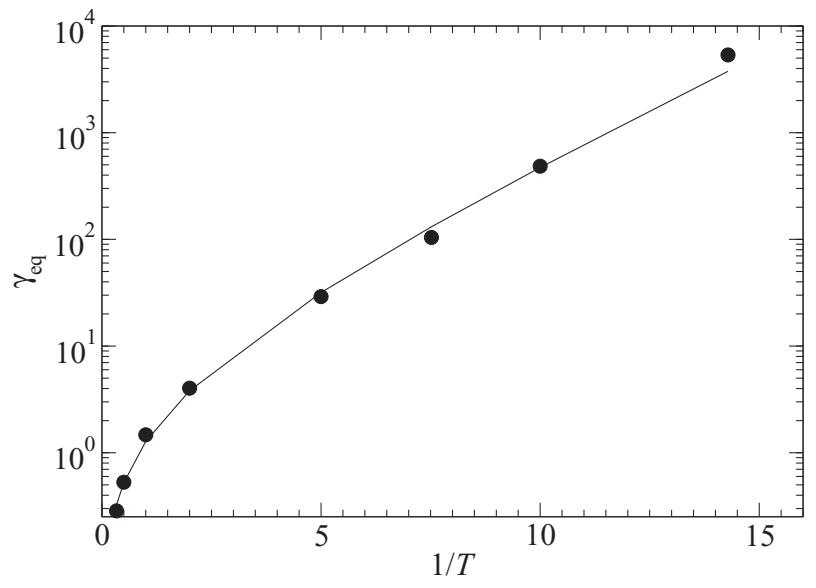

FIG. 9. Equilibrium damping $\gamma_{\mathrm{eq}}$ as a function of inverse temperature $1 / T$. The solid line is a fit with an Eyring equation of the form $\gamma_{\mathrm{eq}}=\beta \tilde{\gamma} \exp (\beta \Delta F)$ with $\tilde{\gamma}=0.85(6)$ and $\Delta F=0.40(1)$.

fixed). The "requested" relative error was varied between $5 \%$ at high $T$ and $0.5 \%$ at low $T$.

From the fits shown in Fig. 8, one can deduce rather accurately the equilibrium damping $\gamma_{\mathrm{eq}}$, which one may loosely associate with an equilibrium viscosity. Results for $\gamma_{\mathrm{eq}}$, see Fig. 9, should be particularly trustworthy when $v$ can be lowered sufficiently much so that $F_{k}(v) \propto v$ can be observed directly, i.e., for $T \geqslant 0.07$. Otherwise, small uncertainties in fitting the coefficient $a_{1}$ can lead to exponentially large errors in $\gamma_{\text {eq. }}$. For $T \geqslant 0.07$, the effective viscosity can be well fit by an Eyring equation.

\section{DISCUSSION AND CONCLUSION}

In this work, numerical solutions to the Langevin equations of the PT model were presented. They covered zero temperature and two decades in finite temperature, as well as up to six decades in sliding velocity at the smallest temperatures. The longest runs at the smallest velocity at a given temperature used to span $10^{10}$ reduced time units, which translates to almost one hour real time when compared to AFM experiments. This made possible to observe directly the crossover from linear Stokes damping or viscous friction to Coulomb or solid friction at some intermediate temperatures. There, the transition could be described by a simple $F(v) \propto \operatorname{arsinh}\left(v / v_{c}\right)$ relation, which contains Stokes and Coulomb friction as limiting cases, and has the proper inversion symmetry with respect to sliding velocity. Although the velocity domain spans a few more decades than other works so far, one cannot exclude the possibility that significant corrections to the leading-order $\operatorname{arsinh}\left(v / v_{c}\right)$-term would be needed if even more decades of slow sliding velocities could be accessed in the simulations at very small $T$. But even if this were the case, it is probably legitimate to say that for any quantitative, practical purpose there is no need to replace the constitutive equations, Eqs. (3) or $(15)$, with one that carries a $\left[\ln \left(v / v_{0}\right)\right]^{2 / 3}$ dependence, in particular as it seems difficult to build the crossover to linear response theory into the latter. For any real application, it seems more practical to add a $\left[\operatorname{arsinh}\left(v / v_{c}\right)\right]^{3}$ term so that the friction velocity dependence can be described accurately.
Moreover, when the PT model is applied to the motion of a tip on two-dimensional, crystalline surfaces, ${ }^{27,28}$ one should expect the parameters $a$ and $v_{c}$ to depend on the sliding direction when a tip is pulled over crystal.

The results presented in this work agree with previous observations in which the $\left[\ln \left(v / v_{0}\right)\right]^{2 / 3}$ dependence either was rejected directly ${ }^{13}$ or could not be convincingly established (at least not to the author of this work) on a single, contiguous data set. ${ }^{11,18,19,29}$ It is nevertheless premature to dismiss the rate theories and their tests. As shown in Appendix B, all directly testable approximations made in the theory are rather reasonable, except near (and of course, above) $v_{0}$, which is precisely the velocity regime in which it would be easiest to discriminate between the exponent $2 / 3$ and an exponent of one. Instead, one should recognize that the scaling and the magnitude (as derived in Appendix A) of the prefactor to the $\ln v$ dependence with respect to temperature is correct. Another important test for the rate theory as applied to the regular PT model is the analysis of the depinning forces, which resulted in a distribution close to the prediction made by the theory. ${ }^{30}$ These tests have been repeated successfully by the author during the preparation of this work.

It is difficult to gauge the implication of the results obtained in this work for generalized PT models, in particular for the two-spring, two-mass generalizations of the PT model. ${ }^{10}$ The latter generalization appears to induce additional velocity regimes, which are not captured by the traditional model. This is somewhat surprising, as the effect of a series coupling of two springs should be describable as a single spring that is damped in terms of a memory functional and that experiences colored, thermal noise. Yet, it has been shown that corrections to transition state theories (similar to the one discussed here) are particularly large when the degree of freedom that is associated with the reaction coordinate has little inertia as compared to the bath variables. ${ }^{31}$ There, the transmission coefficient, which describes deviations from transition state theory, depends roughly logarithmically on the mass of the light (or fast) degree of freedom. Thus, for a gigantic mass mismatch between the two masses, this effect might become ascertainable, although damping and mass affect friction only logarithmically.

We can now certainly see it as established that the prefactor to the $\ln (v)$ friction law for the PT model scales with $T^{2 / 3}$, also given the evidence from previous work, in which the friction velocity relationships obtained at different temperatures had been combined into one single master plot. ${ }^{11,18}$ But what does this mean in practice? Usually, friction is not dry but there is an embedded boundary lubricant in between two rubbing surfaces, which leads to a shear stress that increases linearly with normal stress. ${ }^{32,33}$ Instabilities in that boundary lubricant will then induce not only an even clearer $\ln v$ dependence than the numerical solutions of the PT model, but in addition, they exhibit a linear temperature dependence of the $\ln v$ term $^{34-36}$ which is a rather typical experimental finding for macroscopic friction.

\section{ACKNOWLEDGMENTS}

This work was triggered by Rob Carpick's question if the theoretically predicted $(\ln v)^{2 / 3}$ dependence of friction 
on velocity had ever been confirmed by molecular dynamics simulations. The author thanks Hendrick Hölscher for useful discussions that removed some misunderstandings that the author had regarding some pertinent literature as well as Walter Nadler for introducing him to the XOR shift random number generator.

\section{APPENDIX A: ANALYTICAL CONSIDERATIONS}

At sliding velocities where thermal activation is present but still so weak that the barrier is usually crossed only once, it is possible to estimate the amount with which thermal activation reduces $F_{k}$ as compared to the athermal case. In this appendix, I will outline such a calculation. However, two alterations will be made with respect to previous calculations. First, I will take into consideration that the equilibrium position, to which the atom jumps when it crosses the barrier, depends on the current location of the spring. This affects the amount of energy that is dissipated during the instability. Second, I will use a more general rate equation than the previous works. With this latter change, one can rationalize why it might be difficult to ascertain the proper scaling of friction with velocity.

When calculating the energy that is dissipated during one jump, one needs to know the energy at the beginning of the jump and at the end. The instability starts when the particle crosses the energy barrier at $x_{+}$and it ends when it has settled near the absolute minimum $x_{m}$, which depends on the current position of the spring. In order to calculate these points, one needs to know the extrema of the total potential $V_{t}$ given by

$$
V_{t}(X, x)=\frac{k}{2}(x-X)^{2}+V(x),
$$

where $X=v t$ is the spring position at time $t$ and $V(x)$ is the substrate potential, e.g., $V(x)=\cos (x)$ as considered throughout this work. Some relevant points and energies that will be used in the subsequent treatment are introduced in Fig. 10.

Extrema $x_{\mathrm{e}}$ and also saddlepointssatisfy the equation

$$
k\left(x_{\mathrm{e}}-X\right)+V^{\prime}\left(x_{\mathrm{e}}\right)=0,
$$

which implicitly defines the potentially multivalued function $x_{\mathrm{e}}(X)$. Assume we have solved this equation for $X=X_{0}$. If we now move $X$ back by an infinitesimally small amount $\Delta X$, the previous extremum positions $x_{0}-\Delta x$ can be calculated by a Taylor series expansion of Eq. (A2)

$$
\left[k+V^{\prime \prime}\left(x_{0}\right)-\frac{1}{2} V^{\prime \prime \prime}\left(x_{0}\right) \Delta x\right] \Delta x=k \Delta X .
$$

Thus,

$$
\Delta x= \begin{cases}\mp \sqrt{\frac{-2 k}{V^{\prime \prime \prime}\left(x_{0}\right)} \Delta X} & \text { if } k+V^{\prime \prime}\left(x_{0}\right)=0, \\ \frac{k}{k+V^{\prime \prime}\left(x_{0}\right)} \Delta X, & \text { otherwise. }\end{cases}
$$

The upper line on the right-hand side of the equation describes how a saddlepoint in $V_{t}(x)$ has arisen through the merging of previous $x_{ \pm}$positions. There, we only consider the case $V^{\prime \prime \prime}\left(x_{0}\right)<0$, as it occurs, for example, in the PT model at the instability point $x_{c}$. (Note that $\Delta x$ is subtracted from $x_{0}$ so that $\Delta x_{+}$is negative, while $\Delta x_{-}$is positive, which makes $x_{+}$ appear to the right from $x_{-}$.)

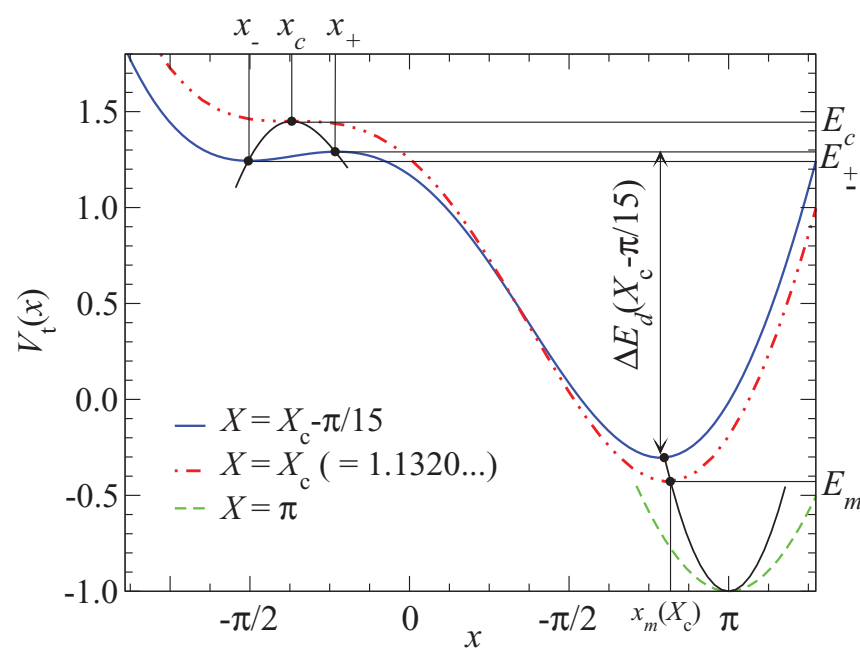

FIG. 10. (Color online) Total potential $V_{t}(X, x)$ for $k=0.4$ as a function of $x$ at two selected spring positions $X$. A few important points are selected and indicated by small, solid circles: The transition point in the athermal case $x_{c}$, the absolute minima for $x_{m}\left(X_{c}\right)$ and $x_{m}\left(X_{c}-\pi / 15\right)$ as well as the relative minimum $x_{-}$and the relative maximum $x_{+}$for $X_{c}-\pi / 15$. Their locations are calculated with the formulas derived in the text.

The saddlepoint itself can be identified by requiring that in addition to $V^{\prime}(x)=0$ also $V_{t}^{\prime \prime}(x)=0$ be satisfied. This imposes the constraint $k+V^{\prime \prime}\left(x_{\mathrm{e}}\right)=0$ or

$$
\begin{aligned}
x_{c} & =\left(V^{\prime \prime}\right)^{-1}(-k) \\
& =-\operatorname{acos}(k) \quad \text { for the PT model. }
\end{aligned}
$$

Here, we have chosen the saddlepoint, where the old metastable site disappears, rather than the one, where a new one arises. Inserting this into $V^{\prime}(x)=0$, the location of the spring during the athermal instability becomes

$$
\begin{aligned}
X_{c} & =x_{c}+\frac{1}{k} V^{\prime}\left[\left(V^{\prime \prime}\right)^{-1}(-k)\right] \\
& =x_{c}+\sqrt{1-k^{2}} / k \text { for the PT model. }
\end{aligned}
$$

In order to calculate the spring position, near which thermal fluctuations usually push the atom over the barrier, we need to know the energy barrier $\Delta E_{b}(X)=E_{+}(X)-E_{-}(X)$. Expressing $V_{t}$ in terms of $\Delta X=X-X_{c}$ and $\Delta x=x-x_{c}$ yields:

$$
E_{ \pm}(\Delta X)=\frac{k}{2}\left(x_{c}-\Delta x_{ \pm}-X_{c}+\Delta X\right)^{2}+V\left(x_{c}+\Delta x_{ \pm}\right)
$$

from where the leading-order approximation

$$
\begin{aligned}
E_{ \pm}(\Delta X)= & V_{t}\left(X_{c}, x_{c}\right)-k\left(x_{c}-\Delta x_{ \pm}-X_{c}\right) \Delta X \\
& +\frac{k}{2} \Delta X^{2}-\frac{V^{\prime \prime \prime}\left(x_{c}\right)}{6} \Delta x_{ \pm}^{3}
\end{aligned}
$$

follows. Note that the last term on the right-hand side of the last equation can be calculated with Eq. (A4) so that the barrier becomes

$$
\Delta E_{b}(\Delta X)=c \Delta X^{3 / 2}
$$


with

$$
\begin{aligned}
c & =\frac{4 \sqrt{2 k^{3}}}{3 \sqrt{-V^{\prime \prime \prime}\left(x_{c}\right)}} \\
& =\frac{4 \sqrt{2 k^{3}}}{3\left(1-k^{2}\right)^{1 / 4}} \text { (for the PT model), }
\end{aligned}
$$

which computes to $c=0.4983$ for $k=0.4$.

To calculate the energy $\Delta E_{d}$ that is dissipated in a jump, we also need to know $E_{m}(X)$. For the calculation of $E_{m}(X)$, it is easiest to take $X=x=\pi$ as the reference point, where $V_{t}(X, x)$ is minimized simultaneously with respect to both its variables. The net potential near this global minimum then simply reads

$$
V_{t}(X, x)=\frac{k}{2}(X-x)^{2}+\cos (x-\pi),
$$

from where

$$
k(X-\pi)=k\left(x_{m}-\pi\right)+\sin \left(x_{m}-\pi\right)
$$

and thus

$$
\left(x_{m}-\pi\right) \approx \frac{k}{1+k}(X-\pi)
$$

follows. Since this equation is linear in $\Delta X$, one can use it in a first approximation not only when $X=\pi$ but also when the reference point is $X=X_{c}$. For the latter, one then obtains

$$
\begin{gathered}
x_{m}(X) \approx \pi+\frac{k}{k+1}(X-\pi), \\
V_{t}\left[X, x_{m}(X)\right] \approx-1+\frac{k}{2(1+k)}(X-\pi)^{2},
\end{gathered}
$$

so that

$$
V_{t}\left(X_{c}-\Delta X\right)-V_{t}\left(X_{c}\right) \approx \frac{k\left(\pi-X_{c}\right)}{1+k} \Delta X .
$$

For the model system investigated here, $k=0.4$ and $\Delta X=$ $\pi / 15$, the derived approximations work very well, as demonstrated in Fig. 10. The most significant error occurs in $x_{m}\left(X_{C}-\pi / 15\right)$ for which the numerically determined value is 2.48 versus the analytically obtained approximation value of $\approx 2.51$. Yet, trends are reflected reasonably well by the leading-order terms for $k=0.4$. For this default value of the spring constant, the time dependence of the energy minimum, which is usually neglected, accounts for about $40 \%$ of the thermal reduction in friction.

If we now knew the average value for $\Delta X=X-X_{c}$, where the instability occurs, we could calculate by what amount $\Delta E_{b}$ thermal fluctuations decrease the energy dissipated during an instability:

$$
\Delta E_{b}=k\left[\left(X_{c}-x_{c}\right)+\frac{\pi-X_{c}}{1+k}\right] \Delta X+\mathcal{O}\left(\Delta X^{3 / 2}\right),
$$

from where we could deduce the thermal reduction in kinetic friction $\Delta F_{k}(T)=F_{k}(0)-F_{k}(T)$ :

$$
\Delta F_{k}(T) \approx k\left[\left(X_{c}-x_{c}\right)+\frac{\pi-X_{c}}{1+k}\right] \frac{\Delta X(T)}{2 \pi},
$$

as in the regime considered here, one instability occurs each time the spring advances by $2 \pi$. This amounts numerically to $\Delta F_{k}(T) \approx 0.237 \Delta X$ for $k=0.4$.
To estimate the population of atoms sitting to the left of the barrier, let us make the usual assumption that the leading-order terms of $V_{t}(x)$ can be obtained by expanding $V_{t}(x)$ into a third-order Taylor series of $x$ around the barrier, which depends explicitly on $X$ and thus implicitly on time. Let us chose the time $t$ such that $X(t=0)=X_{c}$. At small times $t \rightarrow \infty$, the probability $P(t)$ for the atoms to sit left from the barrier equals unity. From then on, the particles can escape the left well. If recrossings are not considered, $P(t)$ is determined by the differential equation

$$
\dot{P}(t)=-w(t) P(t),
$$

where $w(t)$ is the transition probability.

To proceed, one needs a model for $w(t)$. The usual assumption is that a system is always locally close to thermal equilibrium as long as it is driven slowly, in which case $w(t)$ can be expressed as a function of $\Delta X$. Since the Arrhenius law should be obeyed:

$$
w(t)=v \exp \{-\beta \Delta E[\Delta X(t)]\},
$$

where the "attempt frequency" $v$ can still be a slowly varying function of temperature and potentially also of $\Delta X=X-$ $X_{c}$. This latter dependence is usually neglected, but one may assume that the attempt frequency is higher when the distance to crossing the barrier is smaller. Likewise, higher temperatures tend to induce higher attempt frequencies. Since the expectation value of $\Delta X$ increases with $T$, the measured value of $v$ may turn out relatively independent of $T$.

For the just-stated reasons, I will assume the following model for the attempt frequency:

$$
v=v_{0} \frac{k_{B} T}{\Delta X^{\zeta}} .
$$

Here, the dependence on $T$ can be considered to be weak, as the dominant slowing down in the rate when $T$ decreases stems from the exponential factor. Conversely, the dependence of $v$ on $\Delta X$ can become relevant when the athermal instability point is reached, because the rate diverges at $\Delta X \rightarrow 0$ for $\zeta>0$. For $\zeta \geqslant 1$, this would automatically provide the correct boundary condition that $P(t=0)=0$, while the frequentlymade assumption that $v$ is constant violates it in principle.

Following Persson's treatment ${ }^{36}$ the differential equation (A21) can now be solved as usual by

$$
P(t)=\exp \left[-\int_{-\infty}^{t} d t^{\prime} w\left(t^{\prime}\right)\right] .
$$

Since $F_{k}$ depends in leading order on $\langle\Delta X\rangle$, we need to know the average time $\tau$ the atom passes over the barrier. This time can be computed according to

$$
\tau=\int_{-\infty}^{0} d t \dot{P}(t) t .
$$

Introducing $P=1-Q$ and substituting this into Eq. (A24). yields

$$
\begin{aligned}
\tau & =\int_{-\infty}^{0} d t Q(t) \\
& =\int_{-\infty}^{0} d t\left\{1-\exp \left[-\int_{-\infty}^{t} d t^{\prime} w\left(t^{\prime}\right)\right]\right\} .
\end{aligned}
$$


Inspection of the integrand in the exponential function reveals that the inner integrand will crossover from a very small number to a number close to zero in a relatively narrow range. This will happen near a time $t=t^{*}$, where the argument of the exponential function is unity, i.e.,

$$
1=\int_{-\infty}^{t^{*}} d t^{\prime} w\left(t^{\prime}\right)
$$

If we now approximate the main integrand on the right-hand side of Eq. (A27) as zero for $t<t^{*}$ and as unity for $t>t^{*}$, one finds that

$$
\tau \approx t^{*} .
$$

We still need to solve Eq. (A28) for $t^{*}$, i.e., we have to evaluate the integral $I$ on the right-hand side of Eq. (A28). Eliminating $t$ with $\Delta X(t)=-v t$, gives

$$
\begin{aligned}
I & =\int_{-\infty}^{t^{*}} d t^{\prime} w\left[\Delta X\left(t^{\prime}\right)\right] \\
& =\frac{1}{v} \int_{\Delta X^{*}}^{\infty} d(\Delta X) w(\Delta X) \\
& =\frac{v_{0} k_{B} T}{v} \int_{\Delta X^{*}}^{\infty} d(\Delta X) \frac{1}{\Delta X^{\zeta}} e^{-\beta c \Delta X^{3 / 2}},
\end{aligned}
$$

where $c$ can be be ascertained from either equation (A12) or (A13). The integral can now be brought into a simpler form through the substitution $y=\beta c \Delta X^{3 / 2}$ :

$$
I=\tilde{c} \int_{\left(y^{*} / \beta c\right)^{2 / 3}}^{\infty} d y e^{-y} y^{-(1+2 \zeta) / 3}
$$

with

$$
\tilde{c}=\frac{2 v_{0} k_{B} T}{3 v}(\beta c)^{2(\zeta-1) / 3} .
$$

Whenever $(1+2 \zeta) / 3$ is an integer, the integral (A32) reduces to the incomplete gamma function

$$
I=\Gamma\left[\frac{2 \zeta+1}{3},\left(y^{*} / \beta c\right)^{2 / 3}\right],
$$

while in the more general case, where the exponent $(1+2 \zeta) / 3$ in the integral of Eq. (A33) is not an integer, $I$ can be described as a generalization of the incomplete gamma function. The latter can be expanded as a semiconvergent series, which would be sufficiently appropriate for our purposes. It turns out, however, that $\zeta=1$ already produces the desired results, which is why we will only consider this case in the following.

Expanding Eq. (A35) for $\zeta=1$, gives

$$
I=\frac{\tilde{c} \exp \left(-\tilde{y}^{*}\right)}{\tilde{y}^{*}}
$$

with $\tilde{y}^{*}=\left(y^{*} / \beta c\right)^{2 / 3}$. Since $I$ was meant to be unity at $\Delta X^{*}$ and thus at $\tilde{y}^{*}$, we need to solve $1=\tilde{c} \exp \left(-\tilde{y}^{*}\right) / \tilde{y}^{*}$. This can be done by iteration for small values of $\tilde{c}$ through

$$
1=\frac{\tilde{c} \exp \left(-\tilde{y}_{n+1}^{*}\right)}{\tilde{y}_{n}^{*}} .
$$

The first term in the series suffices (with a start value of $\tilde{y}_{0}^{*}=1$ ) when $\tilde{c}$ is very large, which leads to

$$
\tilde{y}^{*} \approx \ln \tilde{c}
$$

for sufficiently large $\tilde{c}$ and thus for sufficiently small velocities. Inserting this result back into the appropriate expressions for $y^{*}$ and $\Delta X$ yields for $\zeta=1$ :

$$
\Delta X \approx\left[\beta c \ln \left(\frac{2 v_{0} k_{B} T}{3 v}\right)\right]^{2 / 3} .
$$

This result is then to be inserted into Eq. (A20). For $k=0.4$, one obtains $a(T) \approx 0.149 T^{2 / 3}$, which represents the line in Fig. 6.

\section{APPENDIX B: NUMERICAL TEST OF ANALYTICAL APPROXIMATIONS}

It is a straightforward task to test numerically the quality of the approximations made in the above treatment. For the case of simplicity, all system variables will be eliminated. In this sense, we define

$$
x^{*}=\int_{-\infty}^{0} d x\left[1-e^{-\tilde{W}(x)}\right]
$$

with

$$
\tilde{W}(x)=\frac{1}{v} \int_{x}^{\infty} d x^{\prime} \tilde{w}\left(x^{\prime}\right)
$$

and

$$
\tilde{w}(x)=\frac{\exp \left(-x^{3 / 2}\right)}{x^{\zeta}} .
$$

The first approximation consists in arguing that

$$
1 \approx \tilde{W}\left(x^{*}\right) \text {. }
$$

The second approximation is composed of two steps: an expansion of $\tilde{W}(x)$ into incomplete gamma functions (where all terms but the first one are discarded, see, e.g., Prandtl's work), followed by an iterative solution of Eq. (B4) that is truncated at zeroth order, resulting in $x^{*} \approx[\ln (v)]^{2 / 3}$. Numerical results for $\zeta=1$ are presented in Fig. 11 .

One can see that the first approximation is rather meaningful, and even the second one still shows the proper trends. Yet,

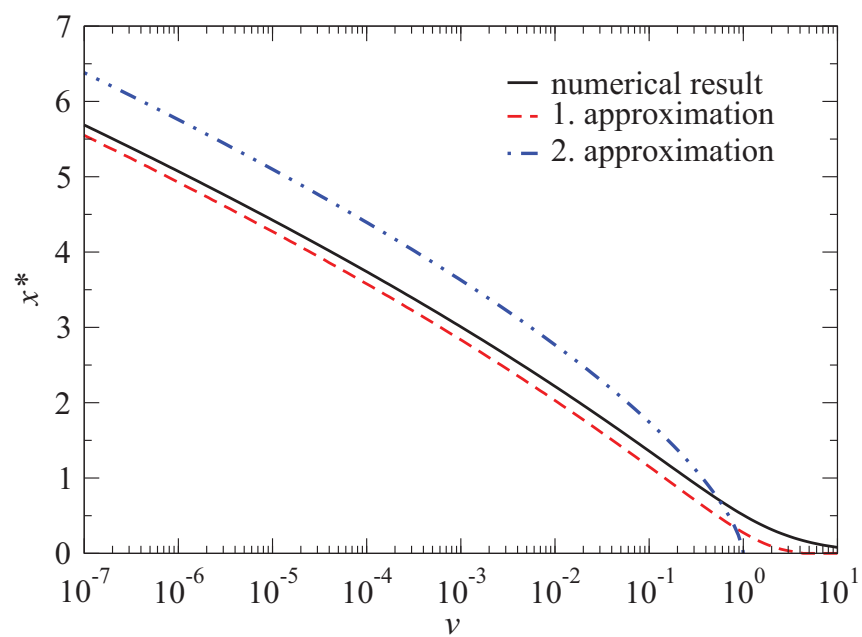

FIG. 11. (Color online) Solutions for $x^{*}$ at different levels of approximation. 
if one were given the numerically accurate solution to $\tilde{x}^{*}$ as an experimental result (which would likely have more scatter and uncertainties than the numerical solutions to the Langevin equation presented in the main part of the manuscript), one should barely be in a position to endorse strongly the $[\ln (v)]^{2 / 3}$ approximation. To do this, one would need to span a few more than seven decades in velocity,at which point $\tilde{x}^{*}$ might be so large that the approximation of the barrier through a cubic polynomial could be at risk or the next order corrections (which happens to be linear in $\ln v$ as one can easily ascertain from Appendix A) starts to kick in.

The shape of the simulated dependence in Fig. 7 is roughly similar to that shown in Fig. 11. They can yet not be mapped onto each other, mainly due to the fact that the athermal $v^{2 / 3}$ contribution also gets reduced because of the thermally-induced premature pops during an instability, and these corrections appear to dominate the friction corrections for the parametrization used in this work.
${ }^{1}$ D. Dowson, History of Tribology (John Wiley, New York, 1998).

${ }^{2}$ L. Prandtl, Z. Angew. Math. Mech. 8, 85 (1928).

${ }^{3}$ G. A. Tomlinson, Philos. Mag. 7, 905 (1929).

${ }^{4}$ D. S. Fisher, Phys. Rev. B 31, 1396 (1985).

${ }^{5}$ E. Gnecco, R. Bennewitz, T. Gyalog, C. Loppacher, M. Bammerlin, E. Meyer, and H. J. Guntherodt, Phys. Rev. Lett. 84, 1172 (2000).

${ }^{6}$ A. Socoliuc, R. Bennewitz, E. Gnecco, and E. Meyer, Phys. Rev. Lett. 92, 134301 (2004).

${ }^{7}$ S. Maier, Y. Sang, T. Filleter, M. Grant, R. Bennewitz, E. Gnecco, and E. Meyer, Phys. Rev. B 72, 245418 (2005).

${ }^{8}$ A. Schirmeisen, L. Jansen, and H. Fuchs, Phys. Rev. B 71, 245403 (2005).

${ }^{9}$ H. Hölscher, A. Schirmeisen, and U. Schwarz, Philos. Trans. R. Soc. A 366, 1383 (2008).

${ }^{10}$ S. Y. Krylov and J. W. M. Frenken, J. Phys. Condens. Matter 20, 354003 (2008).

${ }^{11}$ L. Jansen, H. Hölscher, H. Fuchs, and A. Schirmeisen, Phys. Rev. Lett. 104, 256101 (2010).

${ }^{12}$ M. Evstigneev, A. Schirmeisen, L. Jansen, H. Fuchs, and P. Reimann, Phys. Rev. Lett. 97, 240601 (2006).

${ }^{13}$ O. J. Furlong, S. J. Manzi, V. D. Pereyra, V. Bustos, and W. T. Tysoe, Tribol. Lett. 39, 177 (2010).

${ }^{14}$ P. Reimann and M. Evstigneev, Phys. Rev. Lett. 93, 230802 (2004).

${ }^{15}$ V. L. Popov and J. Gray's translation of Prandtl's work is scheduled to appear in Z. Angew. Math. Mech. in the first issue of 2012.

${ }^{16}$ M. H. Müser, M. Urbakh, and M. O. Robbins, Adv. Chem. Phys. 126, 187 (2003).

${ }^{17}$ Z. P. Yang, H. P. Zhang, and M. Marder, Proc. Natl. Acad. Sci. USA 105, 13264 (2008).
${ }^{18}$ Y. Sang, M. Dube, and M. Grant, Phys. Rev. Lett. 87, 174301 (2001).

${ }^{19}$ O. K. Dudko, A. E. Filippov, J. Klafter, and M. Urbakh, Chem. Phys. Lett. 352, 499 (2002).

${ }^{20}$ B. N. J. Persson, Phys. Rev. B 44, 3277 (1991).

${ }^{21}$ M. Kisiel, E. Gnecco, U. Gysin, L. Marot, S. Rast, and E. Meyer, Nat. Mater. 10, 119 (2011).

${ }^{22}$ S. N. Medyanik, W. K. Liu, I. H. Sung, and R. W. Carpick, Phys. Rev. Lett. 97, 136106 (2006)

${ }^{23}$ S. Y. Krylov, K. B. Jinesh, H. Valk, M. Dienwiebel, and J. W. M. Frenken, Phys. Rev. E 71, 065101(R) (2005).

${ }^{24}$ G. Marsaglia, J. Stat. Softw. 8, 1 (2003).

${ }^{25}$ Q. Y. Li, Y. L. Dong, D. Perez, A. Martini, and R. W. Carpick, Phys. Rev. Lett. 106, 126101 (2011).

${ }^{26}$ M. H. Müser and M. O. Robbins, Phys. Rev. B 61, 2335 (2000).

${ }^{27}$ E. Gnecco, Europhys. Lett. 91, 66008 (2010).

${ }^{28}$ P. Steiner, R. Roth, E. Gnecco, A. Baratoff, and E. Meyer, Phys. Rev. B 82, 205417 (2010).

${ }^{29}$ E. Riedo, E. Gnecco, R. Bennewitz, E. Meyer, and H. Brune, Phys. Rev. Lett. 91, 084502 (2003).

${ }^{30}$ O. K. Dudko, A. E. Filippov, J. Klafter, and M. Urbakh, Proc. Natl. Acad. Sci. USA 100, 11378 (2003).

${ }^{31}$ M. H. Müser and G. Ciccotti, J. Chem. Phys. 103, 4273 (1995).

${ }^{32}$ G. He, M. H. Müser, and M. O. Robbins, Science 284, 1650 (1999).

${ }^{33}$ M. H. Müser, L. Wenning, and M. O. Robbins, Phys. Rev. Lett. 86, 1295 (2001).

${ }^{34}$ G. He and M. O. Robbins, Tribol. Lett. 10, 7 (2001).

${ }^{35}$ M. H. Müser, Phys. Rev. Lett. 89, 224301 (2002).

${ }^{36}$ B. N. J. Persson, O. Albohr, F. Mancosu, V. Peveri, V. Samoilov, and I. M. Sivebaek, Wear 254, 835 (2003). 